\title{
Características de una agencia de comunicación y periodismo digital al servicio de la equidad de género
}

\author{
Characteristics of a Communication and Digital Journalism Agency of the genre equity \\ of service
}

\section{Katherine Seña Giraldo ${ }^{1}$}

Seña G, Katherine

miradas $\mathrm{N}^{\circ} 14$ - 2016. ISSN: 0122 994X Págs 132 - 145

Recepción: Junio 20 de 2016

Aprobación: Octubre 04 de 2016

Publicación: Diciembre 20 de 2016

\section{Resumen}

Este trabajo de investigación determina las características de una agencia de comunicación y periodismo digital al servicio de la equidad de género para crear una agencia de este tipo en Montería (Córdoba, Colombia), aplicando el método investigativo cualitativo exploratorio y la teoría fundada, dado que surgieron categorías emergentes a lo largo del proceso.

La investigación se basó en el estudio de casos tomando a dos agencias, desde dos caracteres, uno social y otro de desarrollo humano: la Fundación Agencia de Comunicaciones Periodismo Aliado de la Niñez, el Desarrollo Social y la InvestigaciónPANDI y la Agencia Comunicación e Información de la Mujer- CIMAC. De igual forma se aplicaron entrevistas estructuradas a expertos académicos y con alta experiencia en esta área. Se analizaron tres categorías a lo largo de la investigación que son: (i) equidad de género y medios de comunicación, (ii) comunicación en nuestra época y (iii) agencia de comunicación y periodismo.

Lo anterior favoreció la formulación de conclusiones, en las cuales se plantea que para contribuir con la equidad de género es necesaria la creación de una agencia de comunicación y periodismo digital que responda a las exigencias de la sociedad de la información y del conocimiento. Una agencia que se caracterice por construir nuevos espacios de participación y cambio social, teniendo como punto de partida indagar las circunstancias en las comunidades objetivo, lo cual podría ser el aliado para conocer las necesidades y potenciar el cambio. Así mismo esto se lograría creando redes interdisciplinarias que posibiliten discusiones de participación colectiva en el tema de equidad de género.

Palabras Clave: Equidad de género, sociedad de la información, sociedad del conocimiento, autocomunicación de masas, participación, agencia de comunicación y periodismo digital.

1 Magíster en Comunicación Digital y Comunicador Social - Periodista. Katherine.sena@upb.edu.co 


\section{Abstract}

This research work determines the characteristics of a Communication and Digital Journalism Agency of the genre equity of service in Monteria, an exploratory qualitative research method and founded theory were applied, since it took place in the practice, where emergent categories emerged.

Theresearch was based on a case study taking two agencies, from two characters, social and human development: La Fundación Agencia de Comunicaciones Periodismo Aliado de la Niñez, el Desarrollo Social y la Investigación-PANDI and Agencia Comunicación e Información de la MujerCIMAC. Similarly structured interviews were applied to high experienced experts in this area. Three categories were analyzed throw-out the research process: (I) gender equality and media, (ii) communication in our time, and (iii) communication and journalism agency.

The foregoing favored the formulation of conclusions, in which it is proposed that to contribute to gender equality it is necessary the creation of a communication agency and digital journalism that meets the requirements of the information and knowledge society. An agency that is characterized by building new spaces for participation and social change, taking as a starting point to investigate the circumstances in the target communities, which could be the ally to meet the needs and promote change. Also this would be achieved by creating interdisciplinary networks that allow collective participation in discussions of the topic of gender equality.

Key Words: Gender equity, information society, knowledge society, mass of auto communication, participation, communications agency and digital journalism.

\section{Introducción}

Para algunos autores, entre ellos Scott (1986), el género es concebido como una expresión que implica hablar de hombres y mujeres como categoría relacional. Scott establece una conexión integral entre dos proposiciones: "el género es un elemento constitutivo de las relaciones sociales basadas en las diferencias que distinguen los sexos y el género es una forma primaria de relaciones significantes de poder" (p. 23).

La equidad de género es entendida desde el Programa de las Naciones Unidas para el Desarrollo-PNUD (2010) como una necesidad para lograr el pleno desarrollo de los derechos humanos, encaminados hacia la búsqueda de la justicia social.

Es así, como se considera que para lograr avances en el tema de equidad de género, se hace necesaria la incorporación de medidas específicas que compensen las desventajas históricas y sociales que involucran a las mujeres.

Según lo descrito por Silva (2008), las Tecnologías de Información y Comunicación-TIC, han representado verdaderamente el primer salto en el dominio del tiempo y del espacio, así como también una revolución tecnológica y social.

Actualmente los medios de comunicación y periodismo digital están optando por estrategias como las agencias de comunicación, con lo cual han experimentado, de una forma especial, todo lo que el periodismo digital ha dispuesto en el mundo de la información actual. Este nuevo recurso implementado por periodistas y comunicadores puede ser utilizado en distintos casos, pero en esta ocasión tendrá relevancia para el tema específico de la equidad de género. 
García (2007) se refirió a este tipo de agencias en el libro Aproximaciones al periodismo digital, de la siguiente forma:

La función de las agencias de comunicación digital como medio al servicio de otros medios se ha incrementado dado que, su papel como proveedor de información textual y gráfica, ha pasado a ser un proveedor de contenidos multimedia, de acuerdo con las necesidades planteadas por las sociedades modernas. (Pág. 101 -102).

Al analizar o intervenir en la relación equidad de género, comunicación $\mathrm{y}$ periodismo digital, es necesario observar su dinámica, puesto que las relaciones de género son específicas de acuerdo con las vivencias de cada país.

Algunos aspectos desiguales sobre equidad de género son evidentes en Colombia, en donde diferentes instancias sociales se han visto afectadas. Por consiguiente, la búsqueda y análisis en el país sobre las estadísticas de género que están evidenciando el grado de inequidad en espacios privados y públicos, materializado en prácticas que muestran la panorámica, tomando como indicadores vivencias y contextos familiares, grados de escolaridad, trabajo remunerado y no remunerado, participación política de las mujeres en comparación con los hombres, relación medios de comunicación y la mujer, entre algunos otros aspectos, los cuales permitirán visibilizar las distintas manifestaciones de las desigualdades de género.

La comunicación y el periodismo digital forman parte de la cadena que afecta la equidad de género, en donde figuran como actores dentro de la problemática social, pero a su vez se presentan también como un mecanismo de ayuda para superar esas mismas dificultades.

Según Esteinou (2002) "la comunicación no es una variable independiente o aislada del desarrollo... de aquí, la importancia vertebral que ocupa actualmente el papel de la producción, difusión, almacenamiento y procesamiento de la información para la superación de los conflictos de nuestra sociedad".

En la actualidad se ha tomado el papel de los medios de comunicación y el periodismo para potencializarlos con el uso de las TIC. De esta forma Carneiro, Toscano y Díaz (2009) manifiestan que "en efecto, ninguna otra tecnología originó tan grandes mutaciones en la sociedad, en la cultura y en la economía. La humanidad viene alterando significativamente los modos de comunicar, de entretener, de trabajar, de negociar, de gobernar y de socializar, sobre la base de la difusión y uso de las TIC a escala global" (p.15).

Como ruta alternativa para esta problemática, desde la comunicación y el periodismo digital, se han venido implementando las llamadas agencias de periodismo digital, cuya ventaja principal según Puerto (2011), es el cubrimiento total de noticias.

Con la implementación de nuevas estrategias, como las agencias de comunicación y periodismo digital, se abre la posibilidad de utilizar las TIC para visibilizar las situaciones de riesgo en que viven mujeres y niñas.

Esta investigación tiene como objetivo determinar las características de una agencia de comunicación y periodismo digital al servicio de la equidad de género y toma como punto de partida el municipio de Montería en el departamento de Córdoba (Colombia), en donde se cuenta, en el ámbito departamental, con la 
Secretaría de Mujer, Género y Desarrollo Social y, en lo municipal, con la Oficina de Participación Comunitaria, como también con la Asesora de Derechos Humanos. En ella se plantea la forma en que la sociedad de la información y del conocimiento a través de las TIC, contribuyen a la participación y la construcción colectiva de conocimiento, lo cual busca propiciar un cambio social desde la equidad de género. Lo anterior se contempla desde procesos de sensibilización que muestren la manera en que hombres y mujeres pueden aprovechar estas herramientas en beneficio propio y común.

Sin embargo, no contemplan en lo referente a periodismo digital estrategias que ayuden o estimulen el posicionamiento de contenidos en la web, en donde se presenten temas de la agenda de género, para que estos dejen de ser asuntos de mujeres, y se puedan evidenciar situaciones de equidad de género.

Por consiguiente, en esta investigación se escogió como instrumento de recolección de información la entrevista estructurada que se aplicó a personas conocedoras del objeto de estudio, por su experiencia en el trabajo con la equidad de género. Así mismo se tomaron dos agencias, con un carácter social y de desarrollo humano.

Estos dos instrumentos se aplicaron teniendo en cuenta tres categorías de estudio que se establecieron desde los objetivos planteados.

- Equidad de género y medios de comunicación

- Comunicación en nuestra época

- Agencias de comunicación y periodismo

Lo anterior soportado en los conceptos que consolidan esta investigación de tipo cualitativo - exploratorio apoyado en la teoría fundada, permitió determinar las características de una agencia de comunicación y periodismo digital al servicio de la equidad de género.

\section{Pregunta de investigación}

¿Cuáles deben ser las características de una agencia de comunicación y periodismo digital al servicio de la equidad de género en Montería, Córdoba (Colombia)?

\section{Materiales y métodos}

De acuerdo con los objetivos formulados se diseñó una entrevista estructurada y se aplicó a las personas conocedoras del objeto de estudio, por su experiencia en el trabajo con la equidad de género.

Así como lo indica Eyssautier (2002):

"El método de entrevista es un intercambio conversacional entre dos o más personas con la finalidad de obtener información, datos o hechos sobre el problema y la hipótesis. En donde señala que la entrevista estructurada es aquella que requiere una serie de preguntas preparadas de antemano por el entrevistador; la entrevista deja la iniciativa total al entrevistado, permitiéndole que se manifieste en forma espontánea" (p. 216).

Por consiguiente se seleccionó este tipo de técnica de recolección de datos, dado que permitió a esta investigación obtener información detallada del objeto de estudio.

Con relación a este, la entrevista tuvo como finalidad obtener información sobre las características que debe tener una agencia de equidad de género de comunicación 
y periodismo digital, así como también conocer información de primera mano de quienes trabajan activamente en el tema de equidad de género.

Para la anterior se tuvieron en cuenta los objetivos específicos:

- Indagar las necesidades comunicativas de los proyectos de trabajo al servicio de la equidad de género.

- Describir los cambios que han surgido en la concepción de una agencia de comunicación y periodismo, a partir de la irrupción de las TIC.

- Identificar las características de una agencia de comunicación y periodismo digital al servicio de la equidad de género.

\section{Muestra para la entrevista}

Para la entrevista se utilizó el muestreo intencional, la muestra estuvo conformada por cinco expertos conocedores de temas de equidad de género y la comunicación digital. Los perfiles requeridos para la selección de los expertos condujeron a la realización de contactos en varios países. Para lo anterior se tuvo en cuenta como criterios de selección el conocimiento y la experiencia acerca del tema que se investiga.

\section{Estudios de casos}

Según Rivera (2006), el estudio de caso es un "examen completo o intenso de una faceta, una cuestión o quizás los acontecimientos que tienen lugar en un marco geográfico a lo largo del tiempo" (Pág.41).

Para este estudio de caso se tomaron dos agencias, con un carácter social y de desarrollo humano: La Fundación Agencia de Comunicaciones Periodismo Aliado de la Niñez, el Desarrollo Social y la Investigación-PANDI y la Agencia Comunicación e Información de la MujerCIMAC. A continuación se hace una breve descripción de cada una de ellas.

La Fundación Agencia de Comunicaciones Periodismo Aliado de la Niñez, el Desarrollo Social y la InvestigaciónPANDI (s.f.).

Es una agencia de comunicaciones que facilita el diálogo entre actores de la sociedad civil, expertos académicos, funcionarios públicos y periodistas, para que los temas relacionados con los derechos humanos, especialmente aquellos que corresponden a los niños, niñas y adolescentes, para tener mayor y mejor cubrimiento, sean prioritarios en la agenda de los medios de comunicación y, a través de ellos, en las políticas públicas formuladas para garantizarlos.

Esta agencia de periodismo y comunicación digitalque trabajapor los temas relacionados con la niñez, la infancia y adolescencia, se escogió teniendo en cuenta que es una de las pioneras en Colombia en agencias digitales con impacto social; además tiene una estructura que sirve como referente o caso de estudio investigativo, para tomarla como ejemplo o guía de las características de una agencia de comunicación y periodismo digital al servicio de la equidad de género en Montería.

Agencia Comunicación e Información de la Mujer-CIMAC (s.f.).

Una organización civil periodística especializada en la perspectiva de género y los derechos humanos, que busca hacer visible la condición social de las mujeres y las niñas, para que, en conjunto con periodistas, organizaciones ciudadanas actoras y actores políticos y la 
academia, contribuyamos a la construcción de un periodismo con perspectiva de género $\mathrm{y}$ una sociedad igualitaria, justa y democrática.

Cuya visión es contribuir con el cambio social y a la democratización de los medios, buscamos influenciar las agendas nacionales y globales a favor de los derechos humanos y la igualdad social.

CIMAC es una agencia líder en trabajos de género desde el periodismo, razón por la cual será tenida en cuenta para el estudio de casos, cuyo propósito es identificar las funciones, las características y la estructura de una agencia de comunicación y periodismo digital al servicio de la equidad de género.

\section{Matriz de análisis de estudio de casos}

Para realizar el análisis de casos se tuvo en cuenta el cuadro de categorías, subcategorías, tópicos y preguntas. De esta forma las mismas preguntas que se aplicaron para la entrevista estructurada, fueron útiles para cuestionar cada uno de los apartes que se requería analizar de las agencias escogidas para efectos del estudio de casos.

\section{Categoría de análisis}

Para la realización de esta investigación se establecieron tres categorías de estudio que parten de los objetivos planteados: equidad de género y medios de comunicación, comunicación en nuestra época y agencias de comunicación y periodismo.

\section{Proceso de investigación y de análisis}

Las actividades realizadas en el proceso de análisis de resultados fueron las siguientes:

Se partió de una primera fase en el cual se realizó el proceso de codificación de las entrevistas.

Primera Fase. Recolección de datos y codificación abierta: en esta fase se aplicaron los instrumentos a los expertos de la muestra, clasificados por ser conocedores en temas de equidad de género y comunicación.

Para la codificación y categorización se realizó un proceso de fragmentación de los datos, el cual permitió observar un conjunto amplio de conceptos ligados a la investigación, y a la vez identificar las categorías donde se muestran aspectos a tener en cuenta para determinar las características de una agencia de comunicación y periodismo digital al servicio de la equidad de género.

Según Soler \& Fernández (2010) la codificación abierta consiste en el análisis involucrado en identificar, nombrar, categorizar y describir los fenómenos encontrados en los datos. Se trata de un proceso analítico donde se identifican las categorías más generales que emergen de los datos brutos, en este seleccionan las palabras claves que generarán los conceptos que serán la base de la próxima fase del proceso, allí se verifican los hallazgos obtenidos en las entrevistas y el estudio de casos, realizando un cruce entre ambos, utilizando las categorías de análisis de las entrevistas como punto de referencia, para ser comparadas con las categorías de análisis del estudio de casos, en los temas que sean posibles.

Es de anotar que la fragmentación de la que se habla está dada por el nivel de 
conocimiento que tienen los expertos sobre cada uno de los puntos tratados en el instrumento.

\section{Resultados}

El objetivo principal de este estudio radicó en recopilar los criterios que permitieran determinar las características de una agencia de comunicación y periodismo digital al servicio de la equidad de género en Montería.

\section{Hallazgos de entrevistas a expertos}

A través de la entrevista se dialogó con los expertos quienes manifestaron que el concepto 'género' lo asocian con la construcción social de la relación hombremujer. Por otra parte al momento de hablar sobre los cambios que pueden presentarse en los conceptos de género y sexo biológico, se encontró que los expertos para este ítem tuvieron variaciones en los dos conceptos indagados. Por una parte, algunos de los expertos afirmaron que ambos conceptos cambian, mientras que otros respondieron que el concepto de género presenta modificaciones.

De las respuestas obtenidas llama la atención las variaciones que se evidencian, esto puede deberse que según Thomas (2010), el concepto de género sufre cambios y modificaciones dependiendo del contexto. Sin embargo, el concepto de sexo biológico no cambia con los procesos académicos o normas jurídicas, mientras el concepto género sí.

Uno de los hallazgos más interesantes es que con respecto a las desigualdades entre hombres y mujeres, todos los expertos coinciden en que estas radican en el aspecto sociocultural y además el experto 01 agregó que “... radica en las posibilidades, en los recursos. Esto afecta a conjuntos de mujeres y hombres que generan diferencia. Genéticamente no somos diferentes. Somos diferentes por las familias y los recursos que cada una tiene.", es decir las desigualdades se encuentran íntimamente ligadas al componente sociocultural.

Acorde a la información obtenida se perfilan como opción, líderes de proyectos de comunicación sobre equidad de género, personas con formación en el tema de equidad de género, independientemente de si pertenecen a la ciudadanía, las mujeres, los medios o los gobiernos.

Al momento de determinar la importancia de la comunicación para la equidad de género se puede llegar a considerar, a partir de las respuestas dadas por los participantes de la investigación, a la comunicación como un proceso valioso en la sociedad, en lo que tiene que ver directamente con la equidad de género. Afirmando que la comunicación juega un papel importante en la forma como se relacionan hombres y mujeres, del cual se dan transformaciones sociales, se construye cultura, se transforman conceptos y se crean enseñanzas, dando lugar a nuevos mensajes y lenguajes.

Se evidenció que en el sistema de comunicación de nuestra época el emisor y receptor se complementan de manera simultánea. Adicionalmente, proporcionan algunas razones por las que se da este fenómeno; algunas de ellas asociadas al surgimiento de redes sociales, dado que el usuario deja a un lado su rol pasivo, para entrar a construir sus propios mensajes, haciendo uso de los medios que ofrece la web.

Otra de las posturas propuestas indica que no se puede desconocer la complejidad del proceso de comunicación y más cuando hay muchos medios que siguen en el funcionamiento tradicional, aún cuando algunos se han abierto a otras posibilidades 
y canales, que en muchas ocasiones no buscan públicos objetivos y no conocen el destinatario final. Esto soporta lo que indica Castells (2009) quien asegura que una de las características de la autocomunicación de masas es la independencia del mensaje.

De allí que se analice en qué sentido se han presentado cambios comunicacionales en la sociedad de la información, a lo cual afirman los entrevistados que la participación es una de las características más notables, dado que los usuarios también crean contenidos.

Al hablar de comunicación actual e interacción oportuna, las respuestas de los entrevistados giran en torno a dos elementos fundamentales, el primero de ellos es el ser humano y el segundo tiene que ver con las TIC. En los planteamientos realizados, la comunicación es un instrumento humano, razón por la cual los principales deben ser los seres humanos, enfatizando sobre mujeres y hombres y luego se incorporan al proceso las tecnologías como instrumentos para llegar a un público específico.

Las respuestas obtenidas en este apartado evidencian que, para los entrevistados hay mucha relación entre la comunicación y el cambio social, incluyendo aspectos a destacar como la construcción de conocimiento colectivo para contribuir al cambio social.

Por consiguiente se cuestiona sobre las consideraciones que se tienen de la comunicación y periodismo digital como una opción para organizar grupos sociales, en donde los expertos manifestaron que es una gran posibilidad porque a través de ellos se pueden mover masas, teniendo en cuenta que las redes sociales siempre han existido y más cuando está latente la necesidad comunicacional. Lo que actualmente permite la comunicación digital y en lo que más contribuye es en la participación que ofrece de manera constante y oportuna para todas las partes involucradas en el proceso.

Se observó que para los expertos la comunicación se define básicamente como un proceso de interacción. Esta dinámica hace que sea necesaria la participación de los miembros de la comunidad y que se generen espacios para la misma. Además, reconocen que si la comunicación se orienta hacia un contexto social específico, es lógico que se trabaje a partir de las necesidades de cada comunidad; y para lograr esta participación es necesario que se reconozcan las intervenciones de los ciudadanos.

Engenerales bien valoradala comunicación interactiva, dada su funcionalidad al proporcionar ventajas de retroalimentación casi instantánea entre emisor y receptores que intercambian información.

El proceso de comunicación si tiene relación con la colaboración de las personas y las instituciones, pero en ella influye el que tiene el poder de organizarla para hacerla más visible.

Los patrones de semejanza obtenidos de las respuestas afirman que la comunicación $\mathrm{y}$ el periodismo digital son herramientas de cooperación, principalmente entre profesionales de la comunicación. Es posible pensar en una sociedad cooperativa, si se tienen en cuenta las posibilidades que brindan los medios digitales en beneficio de los intereses sociales. 
El periodismo según los aspectos a destacar por los participantes del proceso de investigación, ha cambiado con la irrupción de las TIC. Algunos aseguran que las modificaciones a las que ha tenido lugar, están dadas para permitir un contacto con la ciudadanía y obtener mayor retroalimentación de ella. A su vez sugieren que un nuevo medio comunicacional debe hacerse en pro de crear consciencia y responsabilidad social.

Las posibilidades que tendrían los usuarios de una agencia de comunicación y periodismo digital, acorde con las respuestas de los expertos estarían asociadas, principalmente, a la obtención de información, pero esta información debe darse desde distintas miradas contando para ello con fuentes confiables, luego será necesario construir materiales en torno al tema de equidad de género, que sean entendibles y con una muy buena estructura, de manera que quienes tengan acceso puedan comprender y aprender con el uso de los recursos que provee la agencia. Además de lo anterior, se recomienda que el sitio cuente con espacios de participación e interacción para que la agencia sea considerada referente para esta temática.

Dentro de las funciones de una agencia, planteadas por los expertos está hacer el mensaje claro y atractivo para los usuarios, que la información pueda ser comprendida y logre que cada vez más organizaciones de la sociedad civil y personas en general estén involucradas en el tema

A continuación se mencionan las características que debe tener la agencia de comunicación y periodismo digital. Para lo cual los expertos describen el componente informativo, de participación y de interacción como característica que debe estar presente en la organización de la agencia. También "Debe tener como compromiso y como plus principal un carácter formador con los comunicadores y periodistas responsables de la información sobre equidad de género." Así lo aseguró el experto 03 .

Ahora bien, se entiende como una ventaja que la agencia pueda cubrir a profundidad los temas que los medios no pueden, pero no debe convertirse en un espacio que cubra las falencias de otros medios. Por el contrario la agencia deberá suministrar buenos insumos y materiales a quienes necesiten conocer información relacionada con la equidad de género. La agencia debe tener mayor inherencia en los medios de comunicación, pero sin dejar de ser un referente para el resto de la sociedad. Para ello será necesario generar pautas y criterios de organización del conocimiento, que faciliten a las personas acceder a un conocimiento complejo, desde la experiencia cotidiana. La agencia debe depurar la información que entregan las fuentes y reorganizarlas para ganar credibilidad y confianza ante la sociedad.

Toda esta información de la que se nutre la agencia, será comunicada a través de distintos formatos como textos, audios, fotos, videos, infografías y videografías. La variedad y versatilidad establecida, se da en gran medida para captar la atención de usuarios, dependiendo de sus gustos y requerimientos.

Para que la agencia logre realmente la construcción de conocimiento alrededor de la equidad de género, será necesario según expertos: la imparcialidad, generando permanente contacto y diálogo con los diferentes usuarios, manteniendo objetivos claros, y estableciendo un equilibrio entre los retos y los avances.

Otro aspecto a tener en cuenta es la realización de investigación permanente, por parte de miembros responsables, con el fin de construir nuevos contenidos. 
En este orden de ideas, se analiza también como se puede pasar de construir conocimiento a generar participación $\mathbf{y}$ cambio social. Para este ítem se resaltan los aspectos mencionados por el grupo de expertos, quienes afirmaron que se debe partir indagando en la comunidad, para conocer las necesidades, y así potenciar el cambio, otra forma puede ser creando redes interdisciplinarias en las que se promuevan discusiones abiertas, para que exista participación colectiva. Esta misma ejerce su función promoviendo la construcción y discusión desde la sensibilización en el tema de equidad de género.

Ahora bien, los expertos del proyecto, resaltaron en lo que refiere a los cambios de los medios tradicionales a los medios digitales que deben ser cautelosos, puesto que, el tema de la equidad de género no solo se vincula desde la plataforma, sino que se construye desde la conciencia. Para poder efectuar y ver cambios reales, debe existir un empoderamiento más grande por parte de las mujeres.

Los argumentos expuestos durante el desarrollo de esta categoría permiten observar cambios que han surgido en la concepción: agencia de comunicación y periodismo, a partir de la irrupción de las TIC y a su vez se logran identificar las características de una agencia de comunicación y periodismo digital al servicio de la equidad de género.

\section{Hallazgos en el estudio de casos.}

Para el estudio de casos se realizó un cuadro analítico de la caracterización de cada una de las agencias, incluyendo en ellas, algunas subcategorías de las categorías: equidad de género y medios de comunicación, comunicación en nuestra época, y agencias de comunicación y periodismo.
Es de anotar que el estudio de caso se escogió con el objetivo de contrastar la información obtenida en la entrevista con los expertos y el análisis de las agencias CIMAC (Agencia comunicación e información de la mujer) y Pandi Agencia de Comunicaciones Periodismo Aliado de la Niñez, el Desarrollo Social y la Investigación). Teniendo en cuenta que esta última se relaciona con la niñez, no se analizará bajo la categoría equidad de género y medios de comunicación.

Equidad de género y medios de comunicación.

Se toma como punto de partida la relación que hace la agencia CIMAC del concepto género, concebido desde el equilibrio social, pensado principalmente en el fortalecimiento de los derechos de la mujer.

Esta misma agencia perfila como líderes de proyectos de equidad de género a grupos de periodistas, organizaciones ciudadanas, líderes políticos y la academia. Lo anterior, con el objetivo de construir un periodismo que trabaje por el reconocimiento y la búsqueda de una sociedad igualitaria, justa y democrática desde la equidad de género.

En lo que respecta a los temas de equidad de género que han de considerarse importantes para procesos de comunicación. En la agencia se encontró que trabajan las siguientes temáticas: violencia, salud, política, temas laborales, libertad de expresión y derechos humanos, dentro de los cuales trabajan temas específicos como son periodismo y lenguaje no sexista, salud sexual y reproductiva, destruyendo mitos acerca de la violencia, construyendo una cultura de comunicación con equidad, liderazgo de las mujeres, participación política de las mujeres, estrategias de comunicación para organizaciones de la sociedad civil-OSC y cómo estar en los medios sin perdernos. 
Si se contrastan los resultados de las entrevistas con los puntos aquí tratados hay equivalencia entre lo que manifiestan los participantes y lo que se evidencia en las agencias, los expertos indicaron que el género lo asocian con la construcción social de la relación-mujer y en la agencia CIMAC el concepto de género es concebido desde el equilibrio social, en este aparte se puede evidenciar en ambos instrumentos que se hace relevante y pertinente la construcción de equidad social para ambos géneros.

\section{Comunicación en nuestra época.}

De las agencias estudiadas, la agencia PANDI y CIMAC permiten un proceso de interacción con sus usuarios en las de redes sociales como Twitter, Facebook, Flickr Y Youtube. Ofrecen a su vez la posibilidad de suscripción a los boletines que emiten. En este se evidencia, tal como lo indican los expertos que en la comunicación en nuestra época el emisor y el receptor se dan al mismo tiempo. Es decir, lo que han indicado a su vez que la participación de los usuarios es uno de los cambios más notables, dando lugar a que la ciudadanía produzca opinión.

De igual forma así como se verificó en las entrevistas a expertos, la comunicación efectiva logra un cambio significativo socialmente, de esta manera se resalta la labor de las dos agencias, que mediante el uso de herramientas digitales, velan por una comunicación digital más efectiva, que contribuya a que los actores sociales se integren en conjunto con la sociedad, generando cambios y nuevos conocimientos.

Agencias de comunicación y periodismo.

$\mathrm{Si}$ bien es cierto en las agencias convencionales se encuentra información de diversas temáticas para todo tipo de público; sin embargo, las agencias no convencionales así como lo indican los expertos, centran su atención en temáticas específicas y ofrecen al público información de primera mano, organizada y clasificada. De esta forma estas agencias (CIMAC y de PANDI), son referentes de cada una de las temáticas que abordan, dado su trabajo de confiabilidad y credibilidad. Esto se evidencia en las fuentes de información con las que cuentan.

Las agencias de comunicación digital según los expertos entrevistados deben suministrar información en diferentes formatos, dado que la variedad y versatilidad establecida, se da en gran medida para captar la atención de usuarios, dependiendo de sus gustos y requerimientos. Si se contrasta esta información con las agencias estudiadas, se evidencia que PANDI, ofrece a sus usuarios información en textos, videos y fotografías y CIMAC, lo hace con fotos, audios, videos y textos.

\section{Conclusiones}

Luego de haber culminado el proceso investigativo, se concluye que las desigualdades entre hombres y mujeres radican no en el componente genético, sino en el componente sociocultural; para lo cual se hace necesario que los líderes de proyectos de comunicación en equidad de género, sean personas con experiencia en el tema, que orienten desde la comunicación y la educación.

Es importante resaltar que los temas más relevantes a tratar desde la comunicación deben estar relacionados con: políticas públicas, violencia contra la mujer, inequidad femenina, normas que amparan a la mujer, derechos laborales, oportunidades para la mujer en la vida pública, y los derechos sexuales y reproductivos de la mujer. Esto permitiría óptimos resultados sociales desde los nuevos medios comunicacionales de nuestra época, propiciando que las comunicaciones sean 
'multidireccionales' (como la llama Rivas et al, 2009), permitiendo que existan emisores y receptores al mismo tiempo.

$\mathrm{Y}$ es que a la hora de hablar de la comunicación en nuestra época, se evidencia que uno de los mayores pilares es la participación, dado que los usuarios también crean contenidos, gracias a los espacios que se abren a la ciudadanía, en donde producen opiniones y mensajes propios, tal como lo afirma Castell (2009).

Hay que agregar que esto obedece a los cambios de los medios tradicionales a los medios digitales, que han sido asertivos y necesarios, teniendo un gran impacto, no solo en el cambio tecnológico, sino también en el conceptual, en donde se rompe el paradigma de la comunicación vertical y resitúa el papel de la pluralidad de las personas.

Esto lleva a concluir que la participación comunitaria forma parte de una de las características o condiciones que están presentes en los procesos de comunicación para el cambio social.

Con este trabajo de investigación se evidenció que para contribuir con el desarrollo de la temática de la equidad de género se hace necesaria la creación de una agencia de comunicación y periodismo digital, que responda a las exigencias de la sociedad de la información y el conocimiento, debido a que estas posibilitan la obtención de información depurada y de fuentes confiables.

Por consiguiente se busca que, con la creación de la agencia de comunicación se visibilicen proyectos y actividades en beneficio de la equidad de género, y que al mismo tiempo contribuyan con la formación de periodistas desde lo mediático, pedagógico y social. De igual forma la agencia buscará abordar temáticas específicas que vinculen actores sociales con las discusiones colectivas para la generación de nuevo conocimiento.

Así mismo la agencia se debe concebir como una propuesta innovadora, de transformación, y que al mismo tiempo sirva de guía para otros entes, ayudando a la sociedad civil a crear nuevas dinámicas entre hombres y mujeres generando participación y construcción colectiva, dado que cualquiera de estos actores puede ser el creador de una agencia.

Estas funciones se deben enmarcar en unas características claves que son idóneas para una agencia de comunicación y periodismo digital, entre ellas puede destacarse la comunicación, la participación y la interacción, congregando información desde lo social, para luego analizar, contrastar y comunicar.

En lo que corresponde a la agencia, deberá suministrar buenos insumos y materiales a quienes necesiten conocer información relacionada con la equidad de género. Así como también, debe ser un referente para la sociedad, que ayude a la depuración de políticas públicas que beneficien a este sector social. Esto se podría lograr concibiendo pautas y criterios de organización del conocimiento, facilitando que las personas tengan acceso a información compleja, pero desde la experiencia cotidiana.

Al llegar a este punto, es necesario aclarar que los formatos en los que se mostrará la información de la agencia deben responder a las necesidades de los usuarios, es por ello, que se plantea utilizar todos los formatos posibles, entre ellos, textos, audios, fotos, videos, infografías y videografías. Tal como lo indica Alonso (2005) las características anunciadas anteriormente son elementos que integran la nueva comunicación digital, destacando en ello su carácter multimedial, hipertextual e interactivo. 
En este orden de ideas, se concluye que las agencias deben propender por construir conocimiento, generar participación y cambio social, teniendo como primera medida indagar las circunstancias en las comunidades objetivo, lo cual podría ser el aliado para conocer las necesidades, y así potenciar el cambio. Esto también se lograría creando redes interdisciplinarias que posibiliten discusiones de participación colectiva en el tema de equidad de género. Tal como lo propone Levy (2004), quien invita a que se trabaje mancomunadamente para que las personas desde su contexto generen el dinamismo de compartir, transformar constantemente en el mundo digital.

Dentro de este contexto es importante indagar las comunidades a las cuales se va a dirigir o con las que se va a trabajar, para conocer las necesidades de las mismas y generar participación, cambio social contribuyendo con la construcción de nuevo conocimiento.

En coherencia con lo anterior se hace pertinente establecer las características para crear una agencia de comunicación y periodismo digital en la ciudad de Montería, cuyo propósito sea instaurar espacios y priorizar el tema de género en la agenda del Gobierno (local, departamental) y de los medios de comunicación y periodistas de Montería.

Al mismo tiempo esta agencia sentaría un precedente, puesto que su estructura daría parámetros para guiar la forma en que se organiza y distribuye todo lo referente a la equidad de género y de esa forma, atraer la mirada de las autoridades hacia este tema, de modo que se logre un trabajo sustancial y conjunto, donde participen medios de comunicación, periodistas, gobierno local y departamental; y organizaciones que propendan por la equidad de género, y del cual puedan beneficiarse todos.
Para concluir se sugiere la constitución jurídica de la Agencia de Comunicación y Periodismo Digital al servicio de la equidad de género en Montería, bien sea unipersonal o a través de sociedades.

En el curso de la investigación desarrollada quedan como interrogantes que pueden servir para futuras investigaciones como son: ¿Cómo han influido los diferentes cambios de la postmodernidad, la extensión de la Educación y el movimiento de la Mujer? ¿Cuál es la relevancia que se tiene en el tema de la equidad de género en el Departamento de Córdoba? ¿De qué manera ha influido la sociedad de la información y la sociedad del conocimiento en la era digital con relación a la Equidad de género? ¿Cuáles serían los posibles problemas o limitaciones que tiene el funcionamiento de una agencia digital para el fortalecimiento de la equidad de género?

De igual forma se sugiere tener en cuenta temáticas adicionales que surgieron en el proceso de investigación y que no se habían tenido en cuenta al inicio del trabajo. Entre estos se puede destacar la equidad social, el androcentrismo y no androcentrismo, como también la discriminación positiva.

Ante este tipo de estudios es conveniente utilizar metodologías como la etnográfica, estudios dialógicos, narrativas en donde se indague, por medio de entrevistas a profundidad, las experiencias de personas expertas en esta temática.

\section{Referencia bibliográfica}

Carneiro, R., Toscano, J., y Díaz, T. (2009). Los desafíos de las TIC para el cambio educativo. Recuperado de http://www.oei. es/metas2021/LASTIC2.pdf

Castells, M. (2009). Comunicación y poder. Hernández (trad.). (1a Ed.) Alianza Editorial: España 
Esteinou, J. (2002). Los medios de comunicación como instrumentos del Desarrollo. Razón y Palabra. Recuperado de http://www.razonypalabra.org.mx/ anteriores/n29/jesteinou.html

Eyssautier, M. (2002). Metodología de la investigación. (4ta Ed.). México: Ecafsa Thomson learning.

García, A. (2007). Aproximaciones al periodismo digital. Recuperado de http://books.google.com.co/ books?id=7CyTYu7-lcQC\&pg =PA 1 $02 \& 1 p g=$ PA $102 \& d q=$ periodismo + di gital + caso+agencias\&source $=$ bl\&ot $\mathrm{s}=\mathrm{C} 2 \mathrm{hv} 8 \mathrm{QkL} 0 \mathrm{y} \& \operatorname{sig}=\mathrm{i} 3109 \mathrm{yxQdSQ}$ iEA2qYoPiylPZtkE\&hl=es\&sa=X\&- 